\title{
Multiflavor and multiband observations of neutrinos from core collapse supernovae
}

\author{
I. Taboada* \\ School of Physics and Center for Relativistic Astrophysics \\ Georgia Institute of Technology \\ Atlanta, GA 30332. USA
}

(Dated: today)

\begin{abstract}
It has been proposed that the gamma ray burst - supernova connection may manifest itself in a significant fraction of core collapse supernovae possessing mildly relativistic jets with wide opening angles that do not break out of the stellar envelope. Neutrinos would provide proof of the existence of these jets. In the present letter we calculate the event rate of $\gtrsim 100 \mathrm{GeV}$ neutrino-induced cascades in $\mathrm{km}^{3}$ detectors. We also calculate the event rate for $\gtrsim 10 \mathrm{GeV}$ neutrinos of all flavors with the DeepCore low energy extension of IceCube. The added event rate significantly improves the ability of $\mathrm{km}^{3}$ detectors to search for these gamma-ray dark bursts. For a core collapse supernova at $10 \mathrm{Mpc}$ we find $\sim 4$ events expected in DeepCore and $\sim 6$ neutrino-induced cascades in IceCube/KM3Net. Observations at $\gtrsim 10 \mathrm{GeV}$ are mostly sensitive to the pion component of the neutrino production in the choked jet, while the $\gtrsim 100 \mathrm{GeV}$ depends on the kaon component. Finally we discuss extensions of the on-going optical follow-up programs by IceCube and Antares to include neutrinos of all flavors at $\gtrsim 10 \mathrm{GeV}$ and neutrino-induced cascades at $\gtrsim 100 \mathrm{GeV}$ energies.
\end{abstract}

PACS numbers: 96.40.Tv, 97.60.Bw, 98.70.Sa

\section{INTRODUCTION}

Long duration gamma-ray bursts (GRBs) and core collapse supernovae are known to be correlated [1, 2]. $\mathrm{MeV}$ photons that give rise to GRBs are thought to be produced by accelerated electrons in internal shock of jets emitted by the GRB progenitor. Many details about GRB phenomenology remains uncertain. These GRB jets have very narrow opening angles and very large Lorentz boost factors $(\Gamma \gtrsim 300$.) GRBs are one of the plausible candidates sources for the highest energy cosmic rays [3, 4] and as such would observable in $\sim 100 \mathrm{TeV}$ neutrinos by $\mathrm{km}^{3}$ detectors such as IceCube [5] and KM3Net [6].

Only a very small fraction of core collapse supernovae result in GRBs. It has been speculated that the rate of production of jets in core collapse supernovae may be significantly higher than the rate of GRBs, but that often these jets are choked within the supernovae. Evidence for this hypothesis is the observed asymmetry in the explosion of core collapse supernovae 7,8$]$. Very recently evidence for mildly relativistic jets $(\Gamma \sim 1)$ that did break from the progenitor has been presented for type Ic supernovae 2007gr and 2009bb [9, 10]. Supernovae with hidden jets, supernovae with mildly relativistic jets that manage to break out and long GRBs could form a continuum class of astronomical objects. Neutrinos would provide us with information about these hidden jets and early neutrino observations could also lead the way in finding and studying supernovae with mildly relativistic jets that do break out. A model has been proposed by Razzaque, Mészáros and Waxman (henceforth RMW) 11]. This model was

*Electronic address: ignacio.taboada@physics.gatech.edu extended by Ando and Beacom (henceforth $\mathrm{AB}$ ) to include kaon production [12]. The RMW/AB spectrum is very soft leading to $\gtrsim 100 \mathrm{GeV}$ neutrino observations in IceCube/KM3Net. A model for neutrino production in reverse shocks for both choked and successful relativistic jets associated with SN type Ib has also been proposed [13. While we did not consider this latter model in the present letter, the broad conclusions still apply.

A promising way to search for $\gtrsim 100 \mathrm{GeV}$ neutrinos from core collapse supernovae is the optical follow-up [14. Neutrino multiplets in directional and time coincidence trigger observations by fast robotic telescopes. This method has the advantage of being able to dramatically reduce the intrinsic atmospheric neutrino background because the signal search window is only $O(100 \mathrm{~s})$. A neutrino multiplet may not be significant on its own because the accidental rate due to atmospheric neutrino background is $\mathrm{O}(10 /$ year $)$, but the subsequent observation of the rising light-curve of a supernova in spatial and temporal coincidence with a neutrino multiplet would be highly significant. Optical follow-up programs are already in operation by IceCube [15] and Antares [16].

The objective of this letter is to calculate the event rate from core collapse supernovae as observed by $\sim 10 \mathrm{GeV}$ detectors like DeepCore [17]. We propose the optical follow-up programs to be enhanced so as to require at least one $\gtrsim 100 \mathrm{GeV} \nu_{\mu}$ (minimum requirement to achieve good sky localization) and several $\gtrsim 10 \mathrm{GeV}$ events. We also calculate the event rate on $\gtrsim 100 \mathrm{GeV}$ neutrinoinduced cascades. A most interesting case is that of KM3Net, which promises good angular reconstruction of cascades. We propose the optical follow-up programs to be enhanced so as to require at least one $\sim 100 \mathrm{GeV} \nu_{\mu}$ and one $\sim 100 \mathrm{GeV}$ neutrino-induced cascade. The enhanced sensitivity of these two new modes of observation will allow IceCube/KM3Net to provide a more thorough 
test of the RMW/AB model.

\section{NEUTRINO PRODUCTION IN CHOKED JETS}

The RMW/AB model supposes a choked jet with bulk Lorentz boost factor $\Gamma_{b} \sim 3$ and an opening angle $\theta_{j} \sim 0.3$. The kinetic energy of the jet is set to $E_{j}=3 \times 10^{51}$ erg in analogy to GRBs. Also similar to GRBs the time variability of the engine is set to $t_{v} \sim 0.1 \mathrm{~s}$. Neutrinos are produced in p-p interactions via both pions and kaons. The accelerated proton spectrum is assumed to be $d N_{p} / d E=E^{-2}$. The density and energies involved are similar to those of atmospheric neutrinos in which muons do not decay, but instead are subject to radiative cooling. Thus in the case of neutrinos generated by pion decay, the neutrino flavor flux ratio $\phi_{\nu_{e}}: \phi_{\nu_{m} u}: \phi_{\nu_{\tau}}$ is 0:1:0. In the case of kaons there is a small flux of $\nu_{e}$ due to $K_{\mathrm{L}}^{0}$ decay, but the $\mathrm{AB}$ paper does not include neutral kaon contribution. We therefore also assume 0:1:0 as the flavor flux ratio. Taking into account vacuum oscillations the expected flavor flux ratio at Earth is 0.2:0.4:0.4 for both pions and kaons. Note that vacuum oscillations were not taken into account in previous calculations [11, 12], we include them here as they are critical to describe $\nu_{e}$ and $\nu_{\tau}$ fluxes at Earth. All our numbers include vacuum oscillations except where explicitly noted.

The mesons product of $\mathrm{p}$-p interactions have the same initial spectrum as the protons, but they are subject to hadronic and radiative cooling. This results in two break energies above which the meson spectrum is steeper. Neutrinos follow the energy spectrum of their parent mesons. The neutrino flux resulting from pion and kaon contributions can be described as a doubly broken power law:

$$
\frac{d N_{\nu}}{d E}=A\left\{\begin{array}{lr}
E^{-2} & E>E_{\nu}^{(1)} \\
E_{\nu}^{(1)} E^{-3} & E_{\nu}^{(1)} E<E_{\nu}^{(2)} \\
E_{\nu}^{(1)} E_{\nu}^{(2)} E^{-4} & E_{\nu}^{(2)}<E<E_{\max }
\end{array}\right.
$$

$A$ is the flux normalization (for all flavors combined) such that for pions(kaons) $d N_{\nu} / d E$ is $5 \times$ $10^{-2} \mathrm{GeV}^{-1} \cdot \mathrm{cm}^{-2}\left(5 \times 10^{-5} \mathrm{GeV}^{-1} \cdot \mathrm{cm}^{-2}\right)$ at $E_{\nu}^{(1)}$. The energies $E_{\nu}^{(1)}$ and $E_{\nu}^{(2)}$ are the energies above which hadronic and radiative cooling become relevant respectively. For pions (kaons) these energies are: $E_{\nu}^{(1)}=$ $30 \mathrm{GeV}(200 \mathrm{GeV})$ and $E_{\nu}^{(2)}=100 \mathrm{GeV}(20 \mathrm{TeV})$. The values above correspond to a supernova at $10 \mathrm{Mpc}$ with default choices of parameters. See RMW[11] and AB [12] for details.

\section{EFFECTIVE AREAS AND EVENT RATES IN ICECUBE/KM3NET/DEEPCORE}

To calculate the expected number of events $N_{\mathrm{obs}}$ given a neutrino flux $d N_{\nu} / d E$ the neutrino effective area $A_{\text {eff }}$ of a detector is needed:

$$
N_{\mathrm{obs}}=\int d E A_{e f f}(E) \frac{d N_{\nu}}{d E}
$$

The neutrino effective area of IceCube for muon neutrinos averaged over the northern hemisphere and assuming equal $\nu$ and $\bar{\nu}$ fluxes has been published by the IceCube collaboration [18] and it is reproduced in Fig.1. The neutrino effective area of a detector can be calculated from first principles and the procedure for doing so has been reported in many publications. In particular [19] and refs. therein have a careful discussion of this subject.

The calculation of neutrino effective area requires knowledge of the muon effective area, which in turn depends on detailed knowledge of the operation of the detector. In the literature it is common to assume that IceCube has an effective area of $1 \mathrm{~km}^{2}$ for muons above a threshold of $100 \mathrm{GeV}$. This approximation is a good one for the hard spectra, such as that expected from the sources of cosmic rays. In contrast this approximation is a bad one for soft fluxes, such as those from the RMW/AB model. For RMW/AB most of the events observed have energies that are close to the muon detection threshold and thus detailed knowledge of the detector functioning (and hence the muon effective area) are needed. As similar argument also applies to DeepCore and cascade events.

We have used ANIS [20] to simulate neutrinos in the vicinity of IceCube and DeepCore. We assume that IceCube is a cylinder of $1000 \mathrm{~m}$ of height and $564 \mathrm{~m}$ in radius. The center of this cylinder is placed $1950 \mathrm{~m}$ below the ice surface. DeepCore is simulated as a cylinder of $350 \mathrm{~m}$ of height and $125 \mathrm{~m}$ of radius at $2275 \mathrm{~m}$ of depth below the ice surface. The ANIS simulation takes into account the ice/rock boundary, Earth neutrino absorption (though this is a very small effect for the soft RMW/AB spectrum), neutral current regeneration, etc. Our simulations include neutrinos of all flavors. We have assumed flavor flux ratio as appropriate for an the injected fluxes by either pions or kaons and we have taken into account neutrino oscillations. We have also assumed equal ratios of neutrino to anti-neutrino for a given flavor. Muon propagation is calculated with MMC 21]. We assume that events are detected for the following conditions: a) through-going muons or entering muons must have a minimum energy when entering the detector cylinder b) the total visible energy (cascades, muons, etc) of a contained event must be greater than the same threshold as through-going events. For IceCube we have chose $100 \mathrm{GeV}$ as the threshold and for DeepCore $10 \mathrm{GeV}$. IceCube results also apply to KM3Net. We show our calculations for neutrino effective areas for IceCube and DeepCore in Fig. 1. 

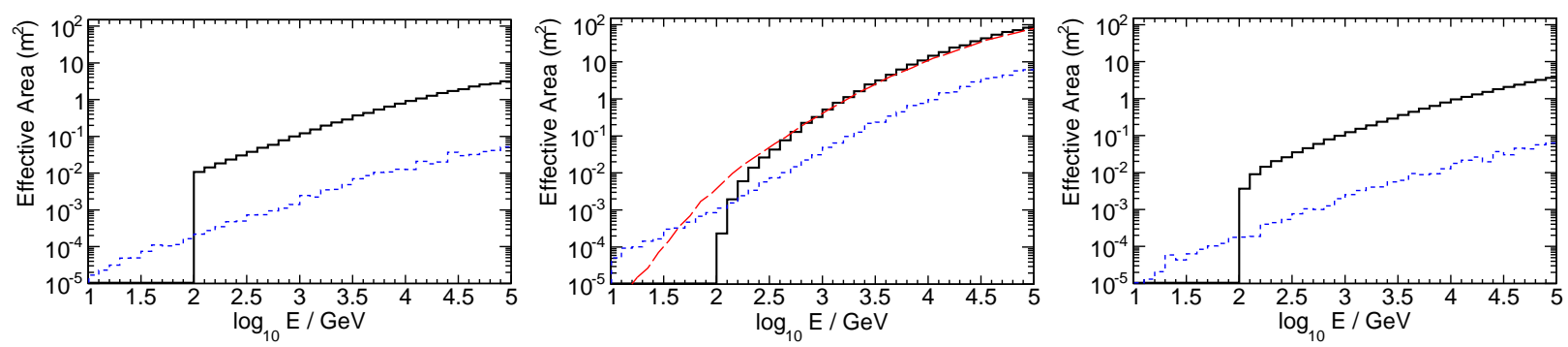

FIG. 1: The three panels show the neutrino effective areas for $\nu_{e}$ (left), $\nu_{\mu}$ (center) and $\nu_{\tau}$ (right). The effective areas shown are for IceCube (solid histogram - black online version) and DeepCore (short dashed histogram - blue online version) as calculated in this letter. Also shown (long dashed curve - red online version) is the effective area for $\nu_{\mu}$ published by the IceCube collaboration. The effective area has been averaged over the northern hemisphere $\left(\delta: 0^{\circ}\right.$ to $\left.90^{\circ}\right)$. The effective area is also averaged over neutrinos and anti-neutrinos. The difference between IceCube's effective area for $n u_{m} u$ as calculated in this letter and as published by the IceCube collaboration is due to threshold effects. In this letter's calculation we have simplistically assumed that all muons with \& $100 \mathrm{GeV}$ energy that go through IceCube are detected. For details see section III.

Note good agreement between our calculations and IceCube published effective for $\gtrsim \mathrm{TeV}$. As described above, for low energies, detailed simulation of the detector performance becomes important. For DeepCore our simulation is in good agreement with the results published by the IceCube collaboration [17]. The IceCube collaboration has not published an effective area for neutrinoinduced cascades. Therefore our results concerning cascades will have the largest uncertainty.

Performing the integral on Eq. 2 using IceCube's published neutrino effective area with the RMW/AB flux for a core collapse supernova at $10 \mathrm{Mpc}$ confirms previous estimates of expected $\nu_{\mu}$ events [12]. Using IceCube' published $\nu_{\mu}$ effective area we obtain an expectation of 11.2 events (28 if we don't assume oscillations as $\mathrm{AB}$ did.) The comparison remains equally valid at high energies for which threshold effects are less relevant. Using our calculation of the $\nu_{\mu}$ effective area we obtain 3.3 events. Our discrepancy with $\mathrm{AB}$ and the IceCube published effective area can be attributed exclusively to threshold effects near $100 \mathrm{GeV}$.

The all-flavor expectation for DeepCore is 4 events. The neutrino-induced cascade signal expected in IceCube is 6.1 events. DeepCore events are broken down by flavor to 0.4 due to $\nu_{e}, 3$ are due to $\nu_{\mu}$ and 0.6 due to $\nu_{\tau}$. Cascade events break down to 2 due to $\nu_{e}, 1.3$ due to $\nu_{\mu}$ (via neutral current interactions) and 2.8 due to $\nu_{\tau}$.

Figure 2 shows the observed event spectra for all three neutrino flavors in DeepCore due to a reference supernova at $10 \mathrm{Mpc}$ that follows the RMW/AB model. Figure 3 is similar but neutrino-induced cascades in a $\mathrm{km}^{3}$ detector. It is clear that the pion component makes the most significant contribution to the observations in DeepCore. In contrast events expected in IceCube, both $\nu_{\mu}$ and cascades, are mostly due to kaons with the exception of energies near to the detector threshold.

\section{ATMOSPHERIC NEUTRINO BACKGROUND}

In DeepCore $10^{5}$ atmospheric neutrinos of all flavors over $2 \pi$ sr are expected per year [17]. IceCube has an atmospheric muon neutrino rate of $10^{5}$ events per year over $2 \pi \mathrm{sr}$ [5]. IceCube has a rate of $2 \times 10^{4}$ atmospheric neutrino-induced cascades per year over $4 \pi$ sr 22].

When searching for neutrino transients, background is significantly reduced by using a narrow time window and by pointing in a specific direction. $\mathrm{Km}^{3}$ neutrino detectors have angular resolutions of $O\left(1^{\circ}\right)$ [30]. Due to the optical properties of ice, IceCube is not expected to be able to reconstruct the direction of cascades well, but it is anticipated that KM3Net will be able to reconstruct cascades with $5^{\circ}$ resolution [23]. It is also expected that DeepCore will reconstruct the direction of events. For charged current $\nu_{\mu}$ events above $\sim 10-20 \mathrm{GeV}$ DeepCore will have a resolution of $10-15^{\circ}$. For cascade-like events in DeepCore the angular resolution is expected to be $\sim 30^{\circ}$ but the minimum energy at which this is feasible is still unknown. DeepCore will also have the ability to separate track events (from C.C. $\nu_{\mu}$ interactions) from cascade-like events for energies greater than $10 \mathrm{GeV}$ [24]. In our calculations below we assume, perhaps pessimistically, an angular resolution of $\sim 30^{\circ}$ for all flavors in DeepCore. The appropriate time search window is set by the size of the star: $\left.R_{*} / c_{\text {light }} \sim 100 \mathrm{~s}\right)$. In fact the radii of progenitors just prior to core collapse are known with poor detail. Candidate progenitor stars have been identified for about a dozen of core collapse supernovae, including SN1987A (type IIP, $R_{*} \sim 10^{11} \mathrm{~cm}$ ) [25] and SN2008D (type $\mathrm{Ib}, R_{*} \sim 10^{11} \mathrm{~cm}$ ) [26]. But SN1987A is unusual and type II progenitors are more frequently red giants with $R_{*}$ as large as $\sim 10^{13} \mathrm{~cm}$.

The rate of accidental atmospheric neutrino multiplets with $N$ events of channel $a\left(\right.$ e.g. $\left.>100 \mathrm{GeV} \nu_{\mu}\right)$ and $M$ (e.g DeepCore) events of channel $b$ is: 

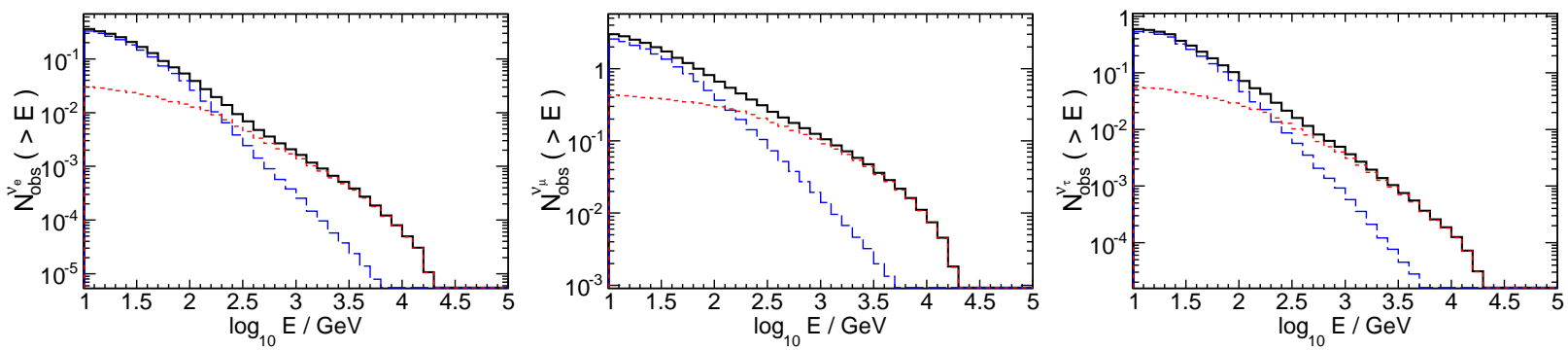

FIG. 2: The panels show the expected signal above a given energy for DeepCore for $\nu_{e}$ (left), $\nu_{\mu}$ (center) and $\nu_{\tau}$ (right). The solid (black in the online version) histogram is the total expectation of the RMW/AB model, the long dashed histogram (blue in the online version) is the pion contribution and the short dashed (red in the online version) histogram is the kaon contribution.
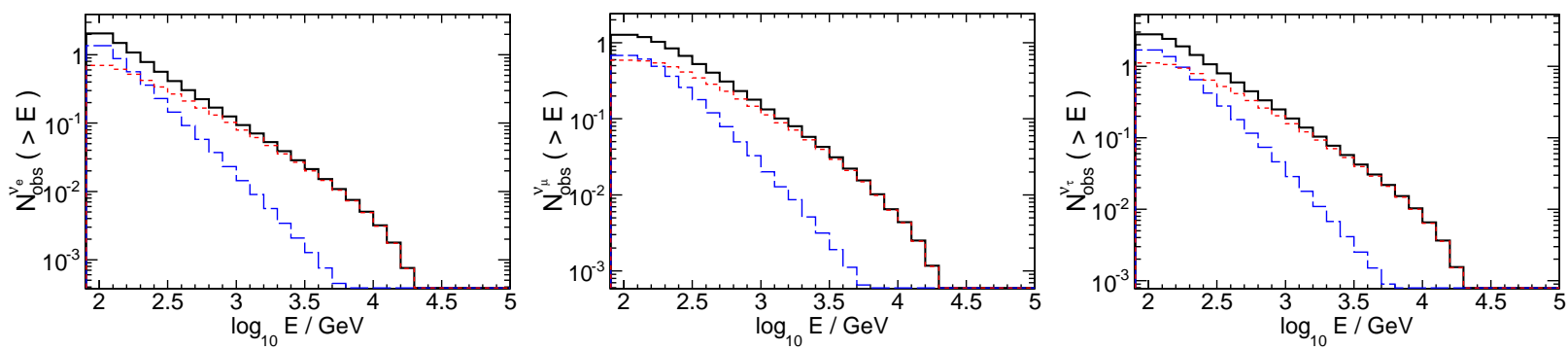

FIG. 3: The panels show the expected signal above a given energy for cascades in IceCube/KM3Net for $\nu_{e}$ (left), $\nu_{\mu}$ (center) and $\nu_{\tau}$ (right). The solid line (black in the online version) is the total expectation of the RMW/AB model, the long dashed line (blue in the online version) is the pion contribution and the short dashed line (red in the online version) is the kaon contribution.

\section{DISCUSSION}

$$
R_{N, M}=R_{a}^{N} R_{b}^{M}\left(\frac{\Omega_{a}}{2 \pi}\right)^{N-1}\left(\frac{\Omega_{b}}{2 \pi}\right)^{M} \frac{\Delta T^{M+N-1}}{(N-1) ! M !}
$$

where $R_{a}$ and $R_{b}$ are the atmospheric neutrino background rates for channels $a$ and $b, \Omega_{a}$ and $\Omega_{b}$ are the angular areas search areas and $\Delta T$ is the temporal search window. Note that eq. 3 is only valid for $N \geq 1$ and $M \geq 0$. In the case that the neutrino multiplet with a single species, it is necessary to set $M=0$.

The rate of accidentals should be matched to at most, what is feasible to follow-up with robotic optical telescopes, about 50/year. The resulting angular uncertainty of the multiband or multiflavor neutrino multiplet must also match the field of view of robotic optical telescopes, about $2^{\circ} \times 2^{\circ}$. Using eq. 3 we find that optical followups are possible for 2 or more $\gtrsim 100 \mathrm{GeV} \nu_{\mu}$ (which has already been proposed), but also (with KM3Net) one $\gtrsim 100 \mathrm{GeV} \nu_{\mu}$ and one $\gtrsim 100 \mathrm{GeV}$ cascade. Two $\gtrsim 100 \mathrm{GeV}$ cascades also produces an accidental rate that might be appropriate, but its follow up would require a very large field of view telescope. Coincidences of one $\gtrsim 100 \mathrm{GeV} \nu_{\mu}$ and at least three DeepCore events also provides a good match to perform optical follow up. Tables I and [I] show the accidental false rate of neutrino multiplets for $\nu_{\mu}$, DeepCore events and cascades.
Following the RMW/AB model, we have calculated the event expectation in DeepCore and for cascades in $\mathrm{km}^{3}$ detectors like IceCube and KM3Net. We find that for a reference core-collapse supernova at $10 \mathrm{Mpc} \sim 4$ events would be detected by DeepCore and $\sim 6$ cascades would be detected by $\mathrm{km}^{3}$ detectors. These signals are strong enough to allow for the search of neutrinos in coincidence with a known supernova in the scale of $10 \mathrm{Mpc}$. In the case of neutrino only searches, the atmospheric neutrino background is higher than what we described in the text, as the time of the explosion can only be established with about 1 day precision [27].

A better alternative is to expand the already running optical follow-up programs. A single $\mathrm{TeV} \nu_{\mu}$ event can provide accurate location in the sky, while a coincident observation with at least three $10 \mathrm{GeV}$ events in DeepCore would result in an acceptable false accidental rate. One muon event and one cascade event at $\gtrsim 100 \mathrm{GeV}$ also has a very low accidental rate in KM3Net, because of its good angular resolution for cascades. A DeepCore-like component in KM3Net may have better angular resolution than in IceCube, but the level of improvement is limited by the kinematical direction difference between the neutrino and the outgoing muon or shower.

The $\gtrsim 10 \mathrm{GeV}$ observations provide another advantage. Because they are sensitive to the pion contribution and the $\gtrsim 100 \mathrm{GeV}$ neutrinos are sensitive to the 
TABLE I: Expected coincident yearly rates of $\mathrm{N} \nu_{\mu}$ events and $\mathrm{M}$ DeepCore events. We assume the $\nu_{\mu}$ events coincident in a circle of $1^{\circ}$ radius and $30^{\circ}$ for DeepCore events. We assume coincidence time window of $100 \mathrm{~s}$.

\begin{tabular}{|c||c|c|c|c|}
\hline \hline $\mathrm{DC} / \nu_{\mu}$ & 0 & 1 & 2 & 3 \\
\hline 0 & - & - & 4.8 & $1.1 \times 10^{-4}$ \\
1 & - & - & 0.2 & $5.0 \times 10^{-6}$ \\
2 & - & 94 & $4.6 \times 10^{-3}$ & $1.1 \times 10^{-3}$ \\
3 & 94 & 1.4 & $6.6 \times 10^{-5}$ & $1.6 \times 10^{-9}$ \\
4 & 1.4 & $1.5 \times 10^{-2}$ & $7.2 \times 10^{-7}$ & $1.7 \times 10^{-11}$ \\
\hline \hline
\end{tabular}

TABLE II: Expected coincident yearly rates of $\mathrm{N} \nu_{\mu}$ events and $\mathrm{M}$ cascade events. We assume the $\nu_{\mu}$ events coincident in a circle of $1^{\circ}$ radius and $5^{\circ}$ for cascade events. We assume coincidence time window of $100 \mathrm{~s}$.

\begin{tabular}{c||c|c|c|c|}
\hline \hline casc $/ \nu_{\mu}$ & 0 & 1 & 2 & 3 \\
\hline 0 & - & - & 4.8 & $1.1 \times 10^{-4}$ \\
1 & - & 12 & $5.8 \times 10^{-4}$ & $1.4 \times 10^{-8}$ \\
2 & 1.2 & $7.3 \times 10^{-3}$ & $3.5 \times 10^{-8}$ & $8.5 \times 10^{-13}$ \\
3 & $7.3 \times 10^{-5}$ & $2.9 \times 10^{-8}$ & $1.4 \times 10^{-12}$ & $3.4 \times 10^{-17}$ \\
\hline \hline
\end{tabular}

kaon contribution, the multiband channel helps the IceCube/DeepCore combination to maintain sensitivity if actual supernovae deviate from the reference model.

Observations of $\gtrsim 10 \mathrm{GeV}$ and $\gtrsim 100 \mathrm{GeV}$ neutrinos in coincidence with core collapse supernovae would be very strong evidence for the existence of choked jets. This observations would help understand the correlation between long duration gamma ray bursts and core collapse supernovae. Finally these observations may provide an alternative way of detecting gamma-ray dark core collapse supernovae with mildly relativistic jets as those observed in SN2007gr and SN2009bb.

The expansion of the optical follow-up programs proposed here is promising in light of the core-collapse supernova rate within $10 \mathrm{Mpc}$ : 1-2 core collapse SNe/yr [28, 29]. The expected opening angle of the choked jets implies a random aligning of one of the jets with the line of sight for $10-20 \%$. This would result in a positive observation every 2.5 - 10 years within $10 \mathrm{Mpc}$ if all core collapse supernovae have hidden jets. But this is a conservative estimation of the relevant supernova rate. The neutrino event rates calculated in this letter imply that observations are possible at distances beyond $10 \mathrm{Mpc}$ and the supernova rate depends of the cube of the distance 31].

\section{Acknowledgments}

We are grateful to John Beacom and Francis Halzen for helpful comments and discussion. We are grateful to Darren Grant for providing information regarding the performance of DeepCore. We thank the anonymous referee for useful comments. This work has been partially supported by National Science Foundation grant PHY0855291.
[1] K.Z. Stanek et al., ApJ 591, L17 (2003)

[2] J. Hjorth et al., Nature 423, 847 (2003)

[3] M. Vietri, ApJ 453, 883 (1995)

[4] E. Waxman and J. Bahcall, Phys. Rev. Lett. 78, 2292 (1997)

[5] J. Ahrens et al. (IceCube collaboration), Astropart. Phys. 20, 507 (2004)

[6] U.F. Katz, Nuc. Inst. Meth. A 567, 457 (2006)

[7] J.R. Maund et al., ApJ. (to be published). Also arXiv:0908.2841

[8] M. Tanaka et al., ApJ 700, 1680 (2009)

[9] Z. Paragi et al., Nature 463, 516 (2010)

[10] A.M. Soderberg et al., Nature 463, 513 (2010)

[11] S. Razzaque, P. Mészáros and E. Waxman, Phys. Rev. D 68, 083001 (2003)

[12] S. Ando and J.F. Beacom, Phys. Rev. Lett. 95, 061103 (2005)

[13] S. Horiuchi and S. Ando, Phys. Rev. D 77, 063007 (2008)

[14] M. Kowalski and A. Mohr A, Astropart. Phys. 27, 533 (2007)

[15] A. Franckowiak et al., in proceedings of the 31st International Cosmic Ray Conference, Lodz, Poland, 2009. Also arXiv:0909.0631

[16] D. Dornic et al., in proceedings of the 31st International Cosmic Ray Conference, Lodz, Poland, 2009. Also
arXiv:0908.0804

[17] C. Wiebusch et al., in proceedings of the 31st International Cosmic Ray Conference, Lodz, Poland, 2009. Also arXiv:0907.2263

[18] A. Achterberg et al., Astropart. Phys. 26, 155 (2006)

[19] M.C. Gonzalez, F. Halzen and S. Mohapatra (unpublished). Also arXiv:0902.1176

[20] A. Gazizov and M. Kowalski, Comput. Phys. Commun. 172, 203 (2005)

[21] D. Chirkin and W. Rohde (unpublished). Also arXiv:hep-ph/0407075

[22] M.V. D'Agostino, Ph.D. thesis, UC-Berkeley, 2009

[23] R. Auer, Nucl. Inst. Meth. A 602, 84 (2009)

[24] D. Grant (private communication)

[25] W. D. Arnett, J. N. Bahcall, R. P. Kirshner and S. .E. Woosley, Ann. Rev. Astron. and Astrophys. 27, 629 (1989)

[26] A. Soderberg et al., Nature 453, 469 (2008)

[27] D.F. Cowen, A. Franckowiak and M. Kowalski, Astropart. Phys. 33, 19 (2009)

[28] M. D. Kistler, H. Yuksel, S. Ando, J. F. Beacom and Y. Suzuki (unpublished). Also arXiv:0810.1959

[29] S. Ando, J. F. Beacom and H. Yuksel, Phys. Rev. Lett. 95, 171101 (2005).

[30] KM3Net may achieve better angular resolution than this 
for higher energy. But at $100 \mathrm{GeV}-1 \mathrm{TeV}$, the angular resolution is dominated by the directional difference between the parent $\nu_{\mu}$ and the daughter muon.
[31] At close distances the detailed distribution of local galaxies is important and does deviate somewhat from the continuous limit 